Meta

Journal des traducteurs

Translators' Journal

\title{
Vers une critique du sens : sémiose en traduction
}

\section{Núria D’Asprer}

Volume 59, numéro 1, avril 2014

URI : https://id.erudit.org/iderudit/1026468ar

DOI : https://doi.org/10.7202/1026468ar

Aller au sommaire du numéro

\section{Éditeur(s)}

Les Presses de l’Université de Montréal

\section{ISSN}

0026-0452 (imprimé)

1492-1421 (numérique)

Découvrir la revue

Citer cet article

D’Asprer, N. (2014). Vers une critique du sens : sémiose en traduction. Meta, 59(1), 8-23. https://doi.org/10.7202/1026468ar

\section{Résumé de l'article}

La question du sens fait l'objet d'un débat traductologique de longue date, mais une problématisation s'avère nécessaire afin de rendre compte de la complexité que le concept recouvre et de ses conséquences sur le plan de la pratique traduisante. En retraçant certaines de ses principales formalisations et en les confrontant à des concepts apparentés, nous souhaitons ouvrir la voie à une réflexion utile au regard du texte, de son interprétation et, a fortiori, de la traduction. Les concepts de sens et de signification, que le langage courant tend à assimiler, sous-tendent pourtant des implications variées, comme en témoignent les nombreuses approches (linguistique, sémantique, sémiotique, psychanalytique), ainsi que les controverses conceptuelles qu'ils ont suscitées. Notre objet n'est pas d'entrer dans le vif de ces débats, mais d'examiner la corrélation entre certaines conceptions du sens héritières du structuralisme et une tendance qui domine le panorama actuel de la traduction. La complexité réelle des textes, la portée symbolique du langage, ce qui se dégage du fait de la polysémie et des textures d'iconicité de l'écriture, nous conduit à accorder la priorité à une conception dynamique du sens. Aussi notre approche de la traduction s'inspirera-t-elle d'une visée non immanente de celui-ci et, plus particulièrement, du concept de sémiose, ou signification en acte, élaboré par Charles Sanders Peirce. Plus pertinente au regard de la signification contextuelle, la productivité signifiante envisagée par Peirce rejoint le concept de chaîne signifiante formalisé par Jacques Lacan : le concept même de signifiant, fondé par le psychanalyste à partir de la désignation saussurienne, mais bien plus proche du signe ou representamen de Peirce, pourrait ouvrir une piste pour explorer la nature complexe des processus de signification et le rapport de ceux-ci avec la traduction. Nos hypothèses seront présentées avec, à l'appui, quelques exemples de traduction (français-espagnol) de textes littéraires modernistes ou post-modernes.
Ce document est protégé par la loi sur le droit d'auteur. L’utilisation des services d’Érudit (y compris la reproduction) est assujettie à sa politique d'utilisation que vous pouvez consulter en ligne.

https://apropos.erudit.org/fr/usagers/politique-dutilisation/ 


\title{
Vers une critique du sens : sémiose en traduction
}

\author{
NÚRIA D'ASPRER \\ Universitat Autònoma de Barcelona, Barcelone, Espagne* \\ nuria.asprer@uab.cat
}

\section{RÉSUMÉ}

La question du sens fait l'objet d'un débat traductologique de longue date, mais une problématisation s'avère nécessaire afin de rendre compte de la complexité que le concept recouvre et de ses conséquences sur le plan de la pratique traduisante. En retraçant certaines de ses principales formalisations et en les confrontant à des concepts apparentés, nous souhaitons ouvrir la voie à une réflexion utile au regard du texte, de son interprétation et, a fortiori, de la traduction. Les concepts de sens et de signification, que le langage courant tend à assimiler, sous-tendent pourtant des implications variées, comme en témoignent les nombreuses approches (linguistique, sémantique, sémiotique, psychanalytique), ainsi que les controverses conceptuelles qu'ils ont suscitées. Notre objet n'est pas d'entrer dans le vif de ces débats, mais d'examiner la corrélation entre certaines conceptions du sens héritières du structuralisme et une tendance qui domine le panorama actuel de la traduction. La complexité réelle des textes, la portée symbolique du langage, ce qui se dégage du fait de la polysémie et des textures d'iconicité de l'écriture, nous conduit à accorder la priorité à une conception dynamique du sens. Aussi notre approche de la traduction s'inspirera-t-elle d'une visée non immanente de celui-ci et, plus particulièrement, du concept de sémiose, ou signification en acte, élaboré par Charles Sanders Peirce. Plus pertinente au regard de la signification contextuelle, la productivité signifiante envisagée par Peirce rejoint le concept de chaîne signifiante formalisé par Jacques Lacan: le concept même de signifiant, fondé par le psychanalyste à partir de la désignation saussurienne, mais bien plus proche du signe ou representamen de Peirce, pourrait ouvrir une piste pour explorer la nature complexe des processus de signification et le rapport de ceux-ci avec la traduction. Nos hypothèses seront présentées avec, à l'appui, quelques exemples de traduction (français-espagnol) de textes littéraires modernistes ou post-modernes.

\section{ABSTRACT}

The problem of meaning has been debated for a long time in Translation Studies, but it seems necessary to reconsider it again in order to display the underlying complexity of the concept and its consequences in the realm of translation practice. Recounting some of its main formalizations and confronting them to related concepts, we hope to foster some useful thinking about text, interpretation, and a fortiori, translation. In fact, the concepts of meaning and signification - that ordinary language tends to collapse into one -, subtend diverse implications, as their many approaches (whether linguistic, semantic, semiotic or psychoanalytic) and the controversies they have given rise to bear witness. It is not our purpose to enter this debate for its own sake, but to examine correlations between some trends inherited from structuralism and the one presently dominant in the field of translation. Actual text complexity, the symbolic bearing of language, all that emanates from polysemy and textures of iconicity in writing, leads us to foreground a dynamic notion of meaning. Our approach of translation will therefore be grounded on a non-immanent purport of meaning, and, more specifically, on the concept of semiosis or signification in action developed by Charles Sanders Peirce. Insofar as it is more relevant with regards to contextual signification, the signifying productivity considered by Peirce comes close to the concept of signifying chain formalized by Jacques Lacan: the 
very concept of signifier, established by the psychoanalyst on the basis of Saussure's terminology, but much more akin to Peirce's sign or representamen, might open a different track to explore the complex nature of signifying processes and the relations of the latter with translation. We support these suggestions with a few examples drawn from translations (French to Spanish) of modernist and postmodern literary texts.

\section{MOTS-CLÉS/KEYWORDS}

sémiose, sens/signification, Charles Sanders Peirce, iconicité, traduction littéraire semiosis, meaning/signification, Charles Sanders Peirce, iconicity, literary translation

La parole n'a jamais un seul sens, [ni] le mot un seul emploi. Toute parole a toujours un au-delà, soutient plusieurs fonctions, enveloppe plusieurs sens. Derrière ce que dit un discours, il y a ce qu'il veut dire, et derrière ce qu'il veut dire, il y a encore un autre vouloir dire, et rien n’en sera jamais épuisé.

(Lacan 1954/1975: 369)

Le travail que nous amorçons ici se déroulera sous l'effet de l'interpellation que provoque le mot sens et, a fortiori, le sens au regard de la traduction.

Notre analyse s'articulera en deux temps: premièrement, une méditation fragmentaire autour du sens, appuyée sur certaines de ses théorisations; deuxièmement, nous présenterons quelques études de cas en traduction littéraire afin d'illustrer les aspects fondamentaux de la problématique abordée.

\section{Parcours critique du sens}

Qu'est-ce que le sens? Que choisit-on en traduction lorsqu'on affirme qu'on traduit le sens? Quel rapport entre sens, signification, signifié?

Tout d'abord, le seul fait d'énoncer le terme sens ferait inévitablement penser au résultat d'un choix dans le binôme forme/sens. La primauté du sens, tout comme la bipartition forme/sens, témoigne d'une conception dualiste du signe issue du Cours de Linguistique générale (CLG), à savoir sa double articulation (Saussure 1916/1985: $\left.97-103^{1}\right)$. Une telle visée débouche souvent sur des interprétations hâtives tendant à associer le signifié au sens et le signifiant à la forme, ainsi qu'à une bipartition tranchée de ces deux faces supposées du signe. Ce n'est pas notre position, mais il est certain que le sens est un concept qui pose problème et que la pratique de la traduction met en lumière une telle problématique.

Le nombre de termes apparentés à sens est révélateur de l'ambiguïté que recouvre celui-ci. Voici, pour l'anglais, sense, meaning, signification, ainsi que le couple signified/signifier, à peu près identique dans les langues où nous œuvrons. Et l'usage souvent aléatoire de ces termes au sein d'énoncés plus ou moins figés témoigne d'un tel flottement et d'un recoupement qui n'est pas identique, ou constant, dans toutes les langues: Traduction et sens, Translation and Meaning, Übersetzung und Bedeutung, Traducción y significado ou Traducción y sentido. Corrélative de la profusion et de la confusion terminologiques que certains sémioticiens ont déjà pertinemment relevées (Eco 1988: 39; Rastier 1990; Hébert 2006), une telle constellation témoigne de différentes manières de concevoir la structure du signe. Si nous attirons l'attention sur elle, c'est parce qu'elle pointe d'emblée vers la problématique du sens et de la traduction. 
Sens et signification sont deux concepts que le langage courant a tendance à assimiler. Or, l'un et l'autre sous-tendent des implications variées, comme le montrent les nombreuses approches (linguistique, sémantique, sémiotique, psychanalytique), ainsi que les confrontations conceptuelles qu'ils ont suscitées.

La question de la signification est une problématique centrale que la tradition philosophique s'est posée de longue date. Aristote avait déjà conçu un triangle sémiotique: parole [1] / concept [2] / chose [3]. Mais à l'époque, l'expérience du sujet individuel n'entrait guère en ligne de compte: si le concept est bien de nature psychologique chez Aristote, il correspond aux états de l'âme, qui sont anthropologiquement partagés.

La parole [1] est un ensemble d'éléments symbolisant les états de l'âme [2], et l'écriture un ensemble d'éléments symbolisant la parole. Et, de même que les hommes n'ont pas tous le même système d'écriture, ils ne parlent pas tous de la même façon. Toutefois, ce que la parole signifie immédiatement, ce sont des états de l'âme qui, eux, sont identiques pour tous les hommes; et ce que ces états de l'âme représentent, ce sont des choses [3], non moins identiques pour tout le monde. (Aristote n. d./1966: 1, 16 a, 3-8)

Il est à noter que chez Aristote, il s'agit d'un enchaînement linéaire, où la diversité de symbolisations dans le langage est liée à l'unicité de sens. Ce n'est que bien plus tard que l'aspect de la connaissance et de l'expérience devait entrer plus pleinement en ligne de compte. Le logicien Gottlob Frege, auteur de Über Sinn und Bedeutung (1891/1971) et inspirateur des sciences du langage, fut sensible à l'ambiguïté du concept de sens et établit une distinction tripartite entre les expressions linguistiques, ce qu'elles signifient (Sinn-sens) et ce à quoi elles réfèrent (Bedeutung ou signification dénotative) (Frege 1891/1971). Il s'agit de la triade: signe, sens et référence, où le sens ( $\underline{\operatorname{Sinn}})$ surgit de l'interaction entre référence et représentation, au lieu d'être un état de l'âme comme c'était le cas chez Aristote. Les états de l'âme, dans la perspective de Frege, correspondraient aux représentations, mais chez lui les représentations sont individuelles (pas universelles). La modernité de Frege en son temps (justement ce qui a été qualifié d'antipsychologisme) fut d'estimer que le sens relevait du langage, de la manière dont il est donné, et non pas de l'intellect ou des processus mentaux de ses usagers, le langage étant considéré par lui comme quelque chose d'objectif tandis que les sens constituent des pensées, car ils émanent du sujet. Voilà donc l'ambiguïté qui surgit de la différenciation fregienne entre représentation (l'élément exclusivement psychologique), sens (siège de l'ambiguïté, à mi-chemin entre l'objectivité du concept et la subjectivité de la représentation) et signification (élément objectif externe ou référent).

Même si actuellement la plupart des langues tendent à assimiler ces deux termes (sens et signification) et à les employer indistinctement, dans le contexte de la traductologie, cette distinction pourrait aider à éclaircir certains points controversés dans le débat sur l'équivalence ${ }^{2}$. On pourrait dire que le sens, dans la perspective de Frege, correspond à une instance interprétative découlant d'une articulation entre signe et référence, autrement dit, à un processus de traduction ${ }^{3}$. Une telle visée ne serait pas loin d'une conception dynamique du sens, car elle suggère déjà un facteur processuel et la mobilité du signe sous l'effet de l'interprétant (instance fondamentale de la sémiotique peircienne à laquelle nous reviendrons). Cette visée complexe des processus de signification inaugurée par les postulats de Frege, surtout la

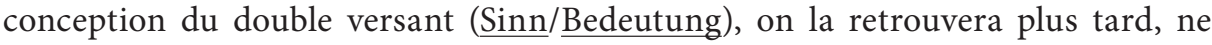


fût-ce qu'implicitement, dans l'articulation par les nouveaux courants linguistiques, principalement pragmaticiens, entre signifié et sens en vertu de la prise en considération du contexte. Nous pensons à la sémantique interprétative de François Rastier (1989), ou à aux approches inspirées par la théorie de la pertinence de Dan Sperber et Deirdre Wilson (1989), et par les travaux sur l'implicite, la polyphonie et l'argumentation d'Oswald Ducrot et de Jean-Claude Anscombre (Ducrot 1984; Ducrot et Anscombre 1983), entre autres. Sur le terrain de la traductologie, c'est sans doute Eugenio Coseriu (1977) qui montre une filiation par rapport à cette distinction fondamentale de Frege entre désignation, signifié et sens: il a bien vu que le sens peut ne pas coïncider avec le signifié ni avec la désignation, car le sens dépend du contexte linguistique et extralinguistique. L'ensemble de ces positions se démarque, certes, du cadre rigide de courants traditionnels qui s'étaient bornés à isoler des unités linguistiques et qui se limitaient au repérage d'unités ultimes de signification (sèmes) dans le mot, où le sens était considéré comme une entité stable et indépendante.

C'est en traversant les frontières disciplinaires que nous découvrons des théorisations où le sens en vient à être déstabilisé. Il en va ainsi de Jacques Lacan (1954/1975; 1966) avec ses conceptualisations princeps, telles que le signifiant ou la chaîne signifiante, auxquelles nous reviendrons. Ou de Deleuze (1969), lorsqu'il pose que le sens est une entité inexistante qui entretient un rapport étroit avec le non-sens. La compréhension du sens chez Deleuze passe forcément par le non-sens. Le non-sens est ce qui, d'après lui, fait fonctionner le sens, ce qui lui permet de circuler (Deleuze 1969: 88). À preuve le fonctionnement des lapsus ou des mots-valises. Lewis Carroll (en littérature) et Magritte (en peinture) nous ont précisément montré à quel point le recours au non-sens est inhérent à la créativité et à la prolifération du sens. Se référant aux mots, Wittgenstein avait affirmé, pour sa part, que ceux-ci n'ont pas de sens, mais des usages (1953/2004). Sans doute anticipait-il sur la pragmatique. Avant lui, Bertrand Russell (1950:3) avait posé l'incapacité des mots à transmettre des signifiés qui ne soient pas enracinés dans une expérience directe et subjective de l'objet du discours. Jakobson, quant à lui, citait précisément cet argument de Russell, qu'il illustrait avec son célèbre exemple du fromage, aboutissant ainsi à une affirmation pertinemment nuancée, d'après laquelle le sens de n'importe quel mot ou groupe de mots est un fait linguistique, mais, plus précisément, un fait sémiotique (Jakobson 1959 [en anglais]; 1959/1963 [en français]). Par «fait sémiotique», il entendait une marge étendue qui dépasse le cadre de la linguistique. Nous avons donc là un tournant fondamental par rapport à la sémiologie saussurienne limitée à la langue. Ainsi, la traduction va être considérée par Jakobson comme un processus de signification (au sens sémiotique) impliquant des signes divers. Autrement dit, il s'agit d'une action sémiotique ou sémiose au sens de Peirce:

Le sens d'un mot n'est rien d'autre que sa traduction par un autre signe qui peut lui être substitué, spécialement par un autre type de signe «dans lequel il se trouve plus complètement développé», comme l'enseigne Peirce, le plus profond investigateur sur l'essence des signes ${ }^{[4]}$. (Jakobson 1959/1963: 79)

En ceci, Jakobson en venait à suggérer que la traduction actualise le sens potentiel des énoncés, que le sens est une instance en devenir.

Sur le terrain de la traductologie, les travaux de François Rastier offrent un apport remarquable. Ils se démarquent nettement des courants linguistiques et traductologiques axés sur le privilège du sens et sur une conception rigide de celui-ci. 
Le linguiste Bernard Pottier s'inscrit dans une telle mouvance, comme l'article au titre révélateur La traduction, une affaire de 'sens' (Pottier 2007) en témoigne. Sur le terrain de la traduction, il en va de même de Charles R. Taber (1972), dont la conception dualiste de la forme et du sens l'avait conduit à envisager le style comme un rajout esthétisant, subsidiaire par rapport au sens, et le sens en tant qu'instance stable à repérer dans les unités lexicales du texte source et à représenter ensuite dans la langue cible de la traduction. L'un comme l'autre semblaient négliger, d'une part, la portée créative du langage et, d'autre part, l'incidence du contexte et des aspects extralinguistiques dans la production de sens. Rastier opérera quant à lui une ouverture par rapport à ce genre de formalisations issues de la linguistique traditionnelle: sensible aux interactions entre signification et sens et à la nécessité d'articuler les acquis des approches grammaticales et stylistiques avec ceux de la philosophie, l'auteur met l'accent sur la situation de communication et envisage une "sémantique textuelle». La traduction, d'après lui, «concerne non les rapports de langue à langue, mais les rapports de texte à texte, puisque tout texte en transforme d'autres» (Rastier $2006^{5}$ ). Une telle prise de conscience de la sémiose nous a paru intéressante, mais le concept de texte semble par ailleurs trop inscrit dans le domaine de la sémiologie linguistique. Sa démarche, inscrite dans les parages de la linguistique, n'a rien de reprochable. Il n'empêche qu'une complexification du concept de texte, étroitement liée à celle du sens, s'avèrerait utile pour aborder la problématique de la signification en traduction. Dans cette perspective, il conviendrait peut-être d'explorer des voies autres que la linguistique.

À ce propos, Roland Barthes, qui a pourtant une ascendance structuraliste, nous propose une piste intéressante lorsqu'il suggère que toute chose peut être texte; bien entendu, il ne s'y réfère pas au sens strict de la linguistique, ni par rapport à une notion rigide et canonique de l'œuvre littéraire, qu'il entreprend de critiquer ici:

[Le texte, dit-il], ce n'est pas un produit esthétique, c'est une pratique signifiante; ce n'est pas une structure, c'est une structuration; ce n'est pas un objet, c'est un travail et un jeu; ce n'est pas un ensemble de signes fermés, doué d'un sens qu'il s'agirait de retrouver, c'est un volume de traces en déplacement; l'instance du texte n'est pas la signification, mais le signifiant, dans l'acception sémiotique et psychanalytique de ce terme [...]. (Barthes 1985: 13)6

Par ailleurs, Barthes envisage une gradation dans le sens. Il y décèle: un niveau informatif, celui de la communication; un niveau symbolique, qu'il rattache à la signification; et un troisième sens plus contingent, qui résiste à la définition et dont le signifié est difficile à saisir: ce troisième sens, il l'appelle signifiance ${ }^{7}$.

En effet, le dernier Barthes (L'Obvie et l'Obtus) s'intéresse de plus en plus à une complexification du sens et envisage un genre d'approche intersémiotique applicable aux arts visuels, mais qui pourrait aussi bien convenir au texte littéraire.

Le sens symbolique s'impose à moi par une double détermination: il est intentionnel (c'est ce qu'a voulu dire l'auteur) et il est prélevé dans une sorte de lexique général, commun, des symboles: c'est un sens qui va au-devant de moi. Je propose d'appeler ce signe complet le sens obvie. Quant à l'autre sens, le troisième, celui qui «vient en trop", comme un supplément que mon intellection ne parvient pas bien à absorber, à la fois têtu et fuyant, lisse et échappé, je propose de l'appeler le sens obtus. (Barthes 1982: 43) 
Cette opposition obvie/obtus est visiblement dérivée de celle qu'il proposait dans $S / Z$ (1970) entre texte lisible et texte scriptible. Par ces oppositions, Barthes semble impliquer une distinction entre un sens référentiel ou dénotatif qui va de soi (la Bedeutung chez Frege) et un sens contingent et fuyant, parfois à la dérive ou en acte: la signifiance (plus proche de ce que Frege entendait par $\underline{\operatorname{Sinn}}$ ). Et nous oserions dire qu'en traduction, la problématique principale, ce qui en fait d'ailleurs l'intérêt, se situe du côté de ce sens obtus. Plus encore, on pourrait soutenir que ce qui la fonde est la confrontation à cette contingence, la traduction étant toujours une expérience qui met en jeu le Sujet, l'incomplétude de celui-ci. Le sens obtus, celui «qui vient en trop ", comme le dit Barthes, et qui peut, en effet, se manifester par défaut, en négatif, active le mouvement de l'interprétation et nous conduit à envisager une analyse du signifiant, de la forme-sens ${ }^{8}$, comme démarche inséparable de la traduction.

Ce sens qui se dérobe, qui reste souvent dans le non-dit ou qui s'inscrit de manière fragmentaire, la signifiance, nous semble essentiel pour aborder la problématique du langage et du sens en traduction et pour justifier une problématisation nécessaire de la vision dichotomique du signe et des oppositions qui en découlent (forme $v s$ sens, expression $v s$ contenu, texte/langue source $v s$ texte/langue cible, etc.). Voilà ce qui nous conduit à considérer le sens comme une instance en devenir; et, par là même, la traduction comme un processus productif de sens qui, à son tour, n'est nullement définitif.

\section{Lacan et Peirce}

Pour éclaircir cette affirmation, il est opportun que l'on examine, ne fût-ce que sommairement, la visée lacanienne quant au sujet et au signifiant en relation avec les concepts de representamen et de sémiose de Peirce9.

Lacan part de la théorie freudienne pour la mener plus loin, et il le fait notamment en l'articulant à la linguistique; mais, par ce même mouvement, il renouvelle aussi certains acquis de la sémiologie. À partir des concepts de métaphore et de métonymie élaborés par Jakobson (1956/1963: 43-67), (associés respectivement aux mécanismes langagiers opérant par similarité - ou paradigmatiques, et à ceux opérant par contiguïté - ou syntagmatiques), il pose que «l'inconscient est structuré comme un langage» et que le sujet de l'énonciation ne coïncide pas avec le sujet de l'énoncé. Le langage comporte une barrière qui sépare le sujet du monde réel. Ce clivage montrant que le vrai sujet est le sujet de l'inconscient, Lacan le représente graphiquement sous la forme d'un Sujet barré, où la barre correspondrait à une faille ou béance.

Postuler un clivage entre énonciation et énoncé, dans le contexte qui nous occupe, pourrait bien être rapproché de l'opposition entre sens et signification étant donné que la signification d'un mot ou d'un énoncé ne correspond pas toujours au sens, qu'elle n'est qu'une partie toute fragmentaire, et parfois différante ${ }^{10}$, du sens qui adviendrait. Ainsi, tout comme le Sujet est marqué par le signifiant, on pourrait dire que les textes sont marqués par le signifiant, barrés et jamais pleinement traduits. Un texte (ou un énoncé ou un mot) peut ne pas être ce qu'il a l'air d'être, seule l'analyse du signifiant, lequel se manifeste souvent sous les textures d'iconicité, peut rendre compte des significations latentes qui continuent d'agir.

La célèbre phrase de Rimbaud «Je est un autre» synthétise assez bien cette réalité du langage, ainsi que le montre le fonctionnement des lapsus ou des actes manqués, 
où certains mots en viennent à en escamoter d'autres ou à les déformer sans que ceux-ci disparaissent complètement pour autant.

D’après Saussure (1916/1985: 98-99), le signifiant est l'image acoustique du signe, pour ainsi dire le côté matériel; dans sa célèbre formule, il plaçait le signifié (Sé) en position prééminente et le Signifiant $(\mathrm{S} a)$ en dessous. Lacan opère une inversion de l'algorithme saussurien, et attribue une primauté au signifiant. Toute structuraliste que cette visée paraisse, par signifiant Lacan traduit le terme Vorstellungsrepräsentanz de Freud, qui désignait le représentant de la représentation. Et il en vient à une définition qui évoque de très près le concept de representamen de Peirce, plutôt que le signifiant de Saussure: «Un signifiant, c'est ce qui représente le sujet pour un autre signifiant» (Lacan 1966: 299).

Selon Peirce,

[un] signe ou representamen est quelque chose qui tient lieu pour quelqu'un de quelque chose sous quelque rapport ou à quelque titre. Il s'adresse à quelqu'un, c'est-à-dire crée dans l'esprit de cette personne un signe équivalent ou peut être un signe plus développé. (Peirce 1897/1978: 121, traduit par Deledalle)

Le signe au sens de Peirce est triadique, il correspond à une action sémiotique:

Un Signe, ou Representamen, est un Premier, qui entretient avec un Second, appelé son Objet, une telle véritable relation triadique qu'il est capable de déterminer un Troisième, appelé son 'Interprétant', pour que celui-ci assume la même relation triadique à l'égard du dit Objet que celle entre le Signe et l'Objet. (Peirce 1897/1978: 147, traduit par Deledalle)

Le signifiant chez Lacan, tout comme le representamen peircien, s'inscrit dans un système triadique dont les catégories sont indissociables. Dans la triade lacanienne, Imaginaire, Symbolique et Réel correspondent en effet aux catégories peirciennes de la priméité, de la secondéité et de la tiercéité ${ }^{11}$.

Notre visée de la signification adhère à la conception dynamique du signe théorisée par Peirce, qui met l'accent sur le signe en acte, le processus sémiotique (ou semiosis) selon lequel tout signe engendre un interprétant qu'il détermine à représenter le même objet que lui, et ce, à l'infini. L'interprétant est donc une instance médiatrice dans le processus de la sémiose. En vertu de ce caractère dynamique, les signes ne sont pas exclusifs, ils s'impliquent entre eux et se transforment donc ${ }^{12}$.

À partir de là, on pourrait envisager une instabilité des textes, y compris, sur le terrain de la traduction, celle du texte dit source. Ceci revient à questionner la notion de parole première ainsi qu'une prétendue relation de subsidiarité du texte cible par rapport au texte de départ. La traduction intervient dans le processus de modification, dans l'actualisation des sens potentiels, qui ne s'épuisent pas. La sémiotique peircienne nous a montré précisément qu’aucun signe n’est définitif, les signes constituant des interprétations de signes préalables. Ubaldo Stecconi (1994) remarquait à juste titre que cela est également applicable dans le domaine de la traduction: les traductions constituent en effet des interprétations de textes antérieurs, et elles suscitent à leur tour de nouvelles interprétations, et ainsi de suite. 


\section{Pratiques traduisantes et sémiose}

Nous avons évoqué précédemment cette faille entre signification et sens, un espace insu qu'il faut traverser, un non-dit à révéler sans l'objectiver. Nous essaierons de montrer, dans cette deuxième partie du travail, l'effet de cette faille ou béance au sein de la sémiose.

Un tel écart se manifeste de manière plus saillante dans la poésie et dans les textes de création en général. Mais la créativité ne doit pas être considérée comme une caractéristique exclusive aux textes littéraires: l'expressivité qui émane de la singularité subjective et de la nécessité d'emprunter des procédés de signification indirects dans toute démarche communicative ne correspond pas à des typologies textuelles particulières. On retrouve ces traits également dans le langage courant. L'exemple le plus remarquable est celui des locutions ou expressions idiomatiques, surtout celles où le principe de fonctionnement est la métaphore; et la métaphore elle-même, qui est à l'origine du langage, repose sur une faille dans la mesure où elle opère un rapprochement analogique de deux termes apparemment divergents à partir d'une comparaison elliptique.

Prenons un exemple, soit faire porter le chapeau (en espagnol, hacer pagar el pato - littéralement: faire payer le [prix du] canard). Naturellement il ne s'agit pas de placer un chapeau sur la tête de qui que ce soit ni, en espagnol, de faire payer le prix d'une volaille, en l'occurrence, le canard (équivalent de pato). Si la traduction mot à mot, qui serait celle du signifié lexical, rend des énoncés grammaticalement admissibles, elle ne correspond pourtant pas au vrai sens de l'expression, lequel ne peut être atteint que si l'on connaît la connotation de l'expression. On pourrait dire qu'il y a eu passage d'une forme analytique (à savoir, la juxtaposition des signifiés lexicaux) à une forme synthétique: la synthèse correspondant au sens de l'expression et qui exige une traversée. Le fait d'employer des termes différents dans chaque langue (chapeau / canard), et sans aucun rapport sémique évident entre eux, nous fait penser à l'arbitrariété (l'une des lois de la sémiologie saussurienne), mais il suffit de retracer l'historique des expressions pour découvrir la motivation de ces signifiants au sein des expressions qui les contiennent. On confirme alors que le sens est contextuel, entendant ici par «contexte» l'historique ou étymologie de l'expression, voire sa sémiose, où le sens s'actualise par analyse, est dégagé par des associations de proche en proche, pouvant parfois être sujet à des variations au fil de l'histoire. Ce processus nous montre que ni chapeau (dans l'expression française) ni pato (dans l'expression espagnole) ne disparaissent complètement, même si la traduction des idiomatismes rend une forme figée, ou plus d'une, selon les sémioses où elles s'inscrivent. Dans l'expression française, connue sous une forme analogue depuis 1669, le chapeau fait référence à celui que les hommes portaient habituellement autrefois, à une époque où l'homme était considéré comme le responsable de la famille. Par association, porter le chapeau est devenu l'équivalent d'avoir la responsabilité. Il a donc fallu passer par une traduction intermédiaire pour en venir à l'actuel sens de l'expression: faire porter la responsabilité d'une erreur à une autre personne pour éviter d'avoir à la prendre soi-même. L'équivalent espagnol hacer pagar el pato s'inscrit dans une sémiose bien différente: la genèse de l'expression nous révèle que pato n'a rien à voir avec l'animal, mais bien avec le mot pacto (pacte) déformé en pato, pacto et Thora étant des termes utilisés par les Juifs espagnols. L’une des interprétations répandues 
soutient que les Juifs, étant le peuple élu, sont obligés de payer un pacte (pacto, en espagnol) avec Dieu lorsqu'ils subissent une calamité. Il en va de même pour bon nombre d'expressions, dont mettre quelqu'un en boîte et son équivalent tomar el pelo a alguien, en l'occurrence, qui ne gardent aucun rapport évident ni avec l'emballage des humains ni avec la coupe des cheveux respectivement.

Le plus souvent, on ignore l'histoire des expressions que l'on utilise à cause de leur ancienneté et, surtout, du fait qu'elles sont lexicalisées et qu'elles fonctionnent comme un tout sémantique unitaire, sourdes aux éléments signifiants originaires, lesquels restent néanmoins latents. En définitive, elles fonctionnent comme la métaphore, et elles révèlent la dimension poétique de l'étymologie ${ }^{13}$.

Les latences que les idiomatismes contiennent, ces espaces non dits par le signifié lexical ni par le sens conventionnel, constituent un espace-charnière, une sorte de texte virtuel; si nous empruntons les termes de Paul Ricœur, une "médiation non verbale» (Ricœur 1975: 271), ou plutôt, non verbalisée, qui se situerait entre le texte de départ et le texte à venir. Un espace en vertu duquel est possible la «comparaison d'incomparables» (Ricoeur 2004: 63), une traduction d'intraduisibles, pourrait-on dire aussi. Il en va de même sur le terrain littéraire et surtout dans la poésie, à cette différence près que les formes d'expression ne sont pas figées ou codifiées sur une base historique, comme dans le cas de nombreux idiomatismes et des proverbes, mais résultent en bonne partie de la créativité, de la singularité du sujet qui les énonce. Le texte poétique opère un rapprochement entre dénotation et connotation de telle sorte que le sens référentiel glisse immédiatement vers la connotation, mais celle-ci ne l'annule point. Peut-être est-ce cela même qui a conduit Noël Audet à affirmer que:

La signification poétique n'est possible que parce que les deux niveaux [sens dénotatif et sens connotatif] fonctionnent simultanément, sinon l'opération de lecture consisterait à traduire littéralement en un langage second ce qui était voilé dans son expression première (le texte), et mieux vaudrait joindre au texte son mode d'emploi, lexique inclus, ou réécrire le poème. (Audet 1978: 460)

Le texte littéraire nous montre donc que la signification est un processus indirect. L’analyse textuelle nous conduit à y déceler l'implicite que les signifiés dénotatifs occultent, ainsi que des réseaux signifiants ou intertextuels subliminaux: tout ce qui se trouve «sous" les mots, nullement subsidiaire et qu'il faudra traduire en empruntant des mécanismes analogues. Un signifiant pourra parfois activer la perception d'une chaîne d'associations inscrite sous la trame textuelle, à savoir une iconicité diagrammatique (Peirce 1897/1978: 149), ce que nous appelons contexte.

Souvent, identifier le contexte dans une unité ou séquence discursive (ou même dans un poème) n'est pas chose facile, et pourtant, cela s'impose ${ }^{14}$. Le contexte peut bien correspondre à cet espace béant que nous avons mentionné, un «texte» intermédiaire à découvrir, sans quoi la traduction pourrait défaillir. Albert Bensoussan l'a très bien montré dans un article où il expliquait la démarche du traducteur aux prises avec ce qu'il appelle «la nuit sombre du sens» (la noche oscura del sentido [Bensoussan 1991]). J'emprunte l'un de ses exemples: cette phrase qui, dans El Beso de la mujer araña (Le baiser de la femme-araignée), de Manuel Puig, est prononcée par son protagoniste, Molina, pour expliquer à son compagnon de prison une séquence filmique: 
(1) Va a la cocina y prepara tostadas, con mantequilla como dicen ellos, y cereales.

(Puig 1976/1988: 2015)

Cet énoncé est apparemment simple à comprendre et une traduction mot-à-mot [elle va à la cuisine et prépare des toasts, avec du beurre comme ils disent, et des céréales] ne choquerait pas si, comme le signale Bensoussan à juste titre, le traducteur n'était alerté par ce syntagme en incise: "y con mantequilla como dicen ellos». Une lecture que nous appellerions sémiosique conduit à y voir, ainsi que le fait Bensoussan, deux contextes simultanés: d'une part, le contexte culturel de l'auteur (et des personnages impliqués dans la situation), l'Argentine, qui nous mène à remarquer qu'à Buenos Aires, pour se référer au beurre, on dirait manteca au lieu de mantequilla, terme habituel en espagnol péninsulaire. Alors, qui dit mantequilla? Qui est ce ellos (du segment como dicen ellos)? Il s'agit du récit d'une scène filmique, et Bensoussan nous rappelle opportunément que le doublage des films américains, à l'époque, était fait par des Mexicains, qui justement emploient aussi ce terme. Ellos, ceux qui disent mantequilla, ce ne sont donc pas les Argentins, mais les Mexicains. Le traducteur, tout comme le lecteur du texte original, est soumis à cette "épreuve de l'étranger" (Berman 1984) qui conditionne la compréhension. Bensoussan y voit un contexte simultané en observant l'usage du suffixe diminutif contenu dans mantequilla, à savoir un signe d'affectation, marque de l'homosexualité que ce personnage, Molina, imprime dans son discours. Cette inscription du sujet est repérée par Bensoussan, qui retrace la trame sémiosique dégagée par l'usage massif des diminutifs dans ce roman, y compris précisément le nom de ce personnage souvent surnommé Molinita par son compagnon de prison. Faute d'un usage maniéré des diminutifs en français, il propose de transposer le code du langage affecté et de traduire en changeant de registre et en privilégiant un autre trait définitoire de Molina, la gloutonnerie, ce qui donne:

(2) Elle va à la cuisine, prépare des toasts, et même du bacon comme ils font, et des céréales.

(Puig 1979/1996: 19, traduit par Albert Bensoussan ${ }^{16}$ )

La marque de l'altérité culturelle que l'usage de mantequilla imprimait dans le texte disparaît au profit d'une autre marque propre à la culture culinaire anglosaxonne, dont les films américains abondent: le bacon. Une telle transformation lexicale évite de tomber dans une tendance annexionniste (Berman 1985) et, en même temps, on pare à l'absurdité de suggérer que les Mexicains puissent dire beurre au lieu de mantequilla dans leurs doublages des films américains en espagnol. Une autre option aurait été de laisser ce mot en espagnol [et avec mantequilla comme ils disent]. Nous serions encline à préférer cette option de non-traduction. Certainement, l'analyse proposée par Bensoussan du diminutif (-illa) par rapport à l'homosexualité de Molina n'est pas anecdotique. À preuve, la surabondance de diminutifs très marquée dans le discours de ce personnage, jointe à l'exaltation généralisée qu'il fait de sa condition sexuelle, ou l'autocomplaisance qu'il montre à se proclamer féminin, d'autant plus que l'un des points forts de cet ouvrage est précisément de mettre en scène la complexité de la position sexuelle du sujet ${ }^{17}$. Mais il conviendrait de remarquer également l'usage massif du diminutif en Amérique latine, indépendamment de toute option sexuelle. Cela dit, il n'est pas étonnant que, finalement, le traducteur ait opté pour substituer bacon à mantequilla. Dans un passage où l'aspect de la 
gloutonnerie prévaut, l'option du bacon est un choix qui se défend. Il s'insère parfaitement dans les paramètres culinaires fournis dans le contexte immédiat, où suivent les crêpes ("y panqueques») parmi d'autres mets.

Le contexte à élucider, cet espace qui lors d'une première lecture semblerait béant, n'est pas toujours d'ordre culturel ou psychologique, comme c'est le cas dans l'exemple que nous venons d'examiner. Il suffit de rester attentif à certains indices pour l'identifier. Il s'agit bien de cette faille entre signifié et sens sur laquelle nous avons insisté. L'analyse textuelle, appliquée à repérer les aspects métatextuels de l'œuvre, ses réseaux signifiants, nous offre la meilleure piste. Et la traduction ne doit pas effacer les pistes, elle ne doit pas chercher à obturer la faille par un recours à la rationalisation ou à l'éclaircissement, lesquelles seraient sans doute des tendances déformantes, comme nous le montrait Berman dans son analytique de la traduction (Berman 1985). Cette faille doit, au contraire, rester ouverte dans le texte d'arrivée, être à même de susciter le parcours sémiosique interprétant que le texte de départ activait. Un tel processus sémiosique confirme la «fonction critique de la traduction» invoquée par Bensoussan (1991) à l'instar de Berman, et surtout il ouvre la voie à une traduction plurielle, empreinte de la marque du traducteur et chaque fois singulière.

La traduction ne saurait se résoudre en adoptant des formes figées supposées équivalentes, mais en dégageant le processus qui est à l'œuvre dans le texte de départ ainsi que les relations et les réseaux signifiants internes, et en tentant d'adopter les mêmes procédés d'écriture dans la traduction. Dans le sillage bermanien, ceci reviendrait à pratiquer une traduction-poésie. Dans cette perspective, l'analyse du signifiant, au sens de Barthes ou de Lacan, nous semble donc prioritaire.

Arrivés à ce point de notre argumentation, il semblerait judicieux de remplacer le concept d'équivalence par celui d'inférence, emprunté à la sémiotique peircienne, dont Ubaldo Stecconi (2001: 17) a tiré un profit tout pertinent. De même que le sens n'est pas un donné existant à priori, le concept d'inférence, contrairement à celui d'équivalence, n'implique pas l'existence à priori d'un équivalent $\mathrm{B}$ (d'un texte A) qu'il s'agirait de rechercher. L'inférence serait quant à elle le processus analytique qui s'instaure par la mise en relation d'une série de facteurs collatéraux, processus en vertu duquel on peut aboutir à produire des équivalents, lesquels peuvent à leur tour en susciter d'autres. L'inférence est donc le processus de recherche qui s'active sous l'effet ou l'interpellation de A, en tant que «fait surprenant». Dans l'exemple que nous venons de présenter, le «fait surprenant», cet énoncé « $y$ con mantequilla como dicen ellos", activait l'analyse et conduisait à inférer un énoncé nullement équivalent en termes linguistiques, mais qui pourtant constitue une interprétation valable: une traduction par inférence.

Voici, pour terminer, un exemple tout différent qui montre l'utilité d'une approche analytique et inférentielle. La problématique porte sur une lettre, $\mathrm{X}$, qui, dans La Guerre, de Jean Marie Le Clézio, a une valeur iconique forte.

Considérons ce passage et la version correspondante, extraite de la seule traduction existante en espagnol:

(3) Quelquefois aussi, il y a, à la terrasse d'un café, au centre de la ville, une jeune fille [...] Elle a des lunettes noires avec des verres bleutés, et de temps en temps, elle relève la tête et elle regarde les gens qui passent. Les éclats étranges sortent de ses lunettes, ils vont droit devant eux, et ils percent de petits trous à la base des colonnes qui soutiennent les prisons, les musées, les banques et les immeubles neufs. Engagez- 
vous dans l'Invincible Armada. Cessez d'être aveugle. Ce sont les yeux qui seront libres les premiers.

Paix.

Bea X.

(Le Clézio 1970: 256 ${ }^{18}$ )

(4) También hay veces, en la terraza de un café del centro de la ciudad, una muchacha. [...] Lleva lentes ahumados con lunas azuladas, y, de tiempo en tiempo, levanta la cabeza y mira a la gente que pasa. Extraños fulgores salen de sus lentes, se proyectan hacia adelante, y agujerean las bases de las columnas que sostienen las prisiones, los museos, los bancos, y los edificios nuevos. Enrólese en la Armada Invencible. Deje de ser ciego. Los ojos serán lo primero que se libere.

Paz.

Bea X.

(Le Clézio 1970/1972: 246, traduit par Hinostroza ${ }^{19}$ )

Faute de pouvoir parcourir le roman dans l'espace limité dont on dispose, il suffira d'indiquer, en abrégeant, que La Guerre est un cas très remarquable sur le plan sémiotique, surtout étant donné la densité de traits iconiques de toute sorte et les rapports complexes que ceux-ci entretiennent. Nous n'analyserons pas exhaustivement le phénomène de l'iconicité multiple, lequel fut l'objet d'un travail précédent $\left(D^{\prime} A s p r e r 2003^{20}\right)$, et nous ne nous attarderons pas, non plus, à dégager les erreurs de traduction évidentes dans ce segment, ni les tournures ou choix lexicaux relevant de la culture latino-américaine du traducteur. Nous porterons attention à ce signe: X.

$\mathrm{X}$ a une double valeur iconique: une iconicité pour ainsi dire conventionnelle, celle qu'implique le symbolisme quasiment universel de ce signe qui, outre une lettre de l'alphabet ou un chiffre romain, dans d'autres contextes représentera une interdiction, un passage barré. Il peut aussi servir à indiquer le danger de telle substance, ou encore une inconnue en mathématiques. X est donc une icône à référents multiples sous-jacents dans l'esprit du lecteur et qui peuvent s'activer au fil de la démarche interprétative. Il suffit, pour saisir une telle densité, que X soit mis en relief, comme c'est le cas dans La Guerre. Dans cet ouvrage, il y a un personnage focal: cette jeune fille du passage examiné, qui s'appelle $B e a B$ en réalité, et qui souvent se promène avec un personnage fruit de son imagination appelé Monsieur X. Elle cherche à déchiffrer les signes de la vie et de la guerre; au fil du récit, elle subit des transformations, elle en exerce aussi. La transformation du nom Bea B en Bea X à laquelle nous assistons ici s'avère donc un indice important de cette transformation et de l'inconnu à traverser, cette béance ou faille de la signification. Le changement d'initiale patronymique dit d'abord deux choses: un "mariage» fantasmé avec Monsieur X, et le passage du début à la fin de l'alphabet, du début à la fin d'une histoire.

Les valeurs symboliques de X pointent d'emblée la transformation, $\mathrm{X}$ étant cette inconnue qu'en mathématiques n'importe quel chiffre pourrait remplacer. X contient donc l'interprétation programmatiquement, c'est un méta-interprétant. Dans ce passage, il est d'autant plus visible qu'il s'insère dans un format iconiquement typifié suggérant la fin d'une lettre: la salutation finale nullement conventionnelle ici (Paix), suivie par la signature (Bea $X)$. Remarquons aussi la disposition en deux lignes parallèles, à la manière des vers dans un poème, ce qui contribue à souligner ce signe par un effet de rime, plutôt visuelle que phonique ici. X s'inscrit donc dans une sémiose au long de cet ouvrage, il fournit un phénomène intertextuel: on le trouve sous la 
forme de ratures, les mots barrés étant fréquents dans La Guerre mais aussi dans Les Géants (1973) ou dans Le Procès verbal (1963) ${ }^{21}$. Dans d'autres cas, le X apparaît doublé: "Cher XX», dit à un moment donné Bea B s'adressant à Monsieur X (Le Clézio 1970: 152; voir note 18); quelques pages plus loin, elle terminait l'une des pseudolettres adressées à Monsieur X en disant «J'aime la paix», et elle signait "XY » au lieu de «Bea B», et ailleurs on avait vu aussi des empreintes pneumatiques coupant le texte et formées par les lettres XIZ alignées (Le Clézio 1970: 115; voir note 18). Il convient de rester attentif à un tel réseau signifiant pour saisir la valeur iconique de $\mathrm{X}$. C'est sous l'action d'un interprétant dynamique, selon la terminologie de Peirce, qu'un tel processus sémiosique s'active.

Quelles conséquences sont à tirer dans le passage qui nous occupe? Ni traduction cibliste ni sourcière, mais il s'agira de porter une attention extrême à ce que le texte réclame de par sa signifiance. Paix ne peut pas admettre l'équivalent immédiat $\mathbf{P a z}$ comme traduction, car il faut préserver le X. C'est ainsi qu'au cours de nos séminaires de traduction nous avions suggéré le latinisme Pax (D’Asprer 2003 : 101). Sans doute on dira qu'il brise l'unité linguistique. Une licence? Nullement, étant donné que dans La Guerre abondent les discontinuités de tout ordre, y compris des énoncés ou des mots en langues étrangères, ainsi que quelques mots en latin qui choquent par rapport au continuum discursif caractérisé par un registre standard et une syntaxe assez simple. Ce choix, qui déjà se soutenait par les constatations mentionnées ci-dessus, nous a conduit à découvrir ultérieurement que le mot $\boldsymbol{P a x}$ apparaît textuellement dans la Guerre, même si nous ne l'avions pas remarqué parce qu'il est entassé dans l'une des nombreuses listes d'objets, celle qui fournit iconiquement les allées d'un supermarché (concrètement, une liste de produits détergents, p. 232). Le symbolisme de ce X escamoté par la marque de produit chosifiée en $\boldsymbol{P a x}$ en vient à s'actualiser par notre traduction de paix. Ce choix aura, bien entendu, des conséquences pour ce qui est de la traduction d'autres passages du texte, que nous n'aborderons pas ici.

La présence de $\mathrm{X}$, qui, à une première lecture, aurait pu être invisible, est pourtant aveuglante. Bea $\mathrm{B}$ possédait déjà en quelque sorte le $\mathrm{X}$, voilé derrière son nom (comme celui de Beatrix, la Béate et guide de Dante dans son chemin vers le paradis). Pour le savoir, il aurait suffi d'obéir à l'interpellation qu'elle fait au lecteur anonyme qu'incarne Monsieur X: "Cessez d'être aveugle, les yeux seront libres les premiers». Il aurait donc suffi d'ôter ce «voile» de la référentialité qui empêche de voir le signifiant.

(4) Pax

Bea X

Les exemples que nous venons de présenter ne sont pas des cas isolés, mais ils nous ont permis, néanmoins, de remarquer que les textes ne constituent pas des entités stables et homogènes, mais un espace d'ouverture marqué par le signifiant. Le texte à traduire serait, comme l'a observé Fernand Verhesen à propos de la poésie, l'étape d'un cheminement (Verhesen 2003: 7), «un phénomène en phase d'instabilité» (Garelli 1991: 133), pour ainsi dire, un processus que la traduction serait à même de développer. Cela explique, par conséquent, la nécessité de porter une attention toute particulière aux enjeux de l'écriture et d'adopter une attitude analytique et agissante au sein de cette ouverture que le texte déploie en sa sémiose. La tendance dominante sur le terrain de la traduction, trop acharnée à rendre un supposé sens à partir de la référentialité, débouche souvent sur des textes neutralisés et dont le fonc- 
tionnement ne fait pas jouer des modes de lecture ni des stimulations homologues en langue cible. De tels procédés conduisent à effacer l'hétérogénéité du langage et du discours et risquent d'entraîner une clôture du sens. La traduction est forcément soumise à la contingence de la signification, mais elle l'est précisément à cause de la différence, cette faille que nous avons essayé de définir au fil de notre réflexion.

\section{NOTES}

* Ce travail s'inscrit dans une recherche individuelle réalisée au sein du groupe Étienne Dolet d'études transdisciplinaires en traduction et du groupe TransMedia (code 2009SGR700) de l'Université Autonome de Barcelone.

1. Remarquons que sens et signification ne sont pas des concepts déterminants chez Saussure, du moins si on s'en tient à ses travaux autographes, rédigés à partir de 1893 et découverts en 1996: «[...] valeur exprime mieux que tout autre mot l'essence du fait, qui est aussi l'essence de la langue, à savoir qu'une forme ne signifie pas mais vaut: là est le point cardinal. Elle vaut, par conséquent elle implique l'existence d'autres valeurs» (Saussure 1891/2002: 27).

2. Pour une vue d'ensemble rigoureuse et non simpliste des théorisations sur l'équivalence, voir Pym (2010: 6-38).

3. Notons toutefois que différents philosophes contemporains (Chalmers 2006, Brogaard 2007, entre autres), en opposition à Frege, se sont ingéniés à développer des sémantiques bidimensionnelles.

4. Notre note [4] est en fait la note 1 dans l'original. Voir Dewey (1946), cité par Jakobson (1959/1963: 179 , note 1$)$.

5. Ces arguments font penser au concept de sémiose illimitée fondé par Charles S. Peirce. Néanmoins, Rastier définit plus loin la sémiosis textuelle comme «le mode d'appariement spécifique entre le plan de l'expression et le plan du contenu d'un texte» (Rastier 2006: 40).

6. Le texte est d'abord paru dans Le Monde en 1974, puis a été reproduit dans L'Aventure sémiologique (Barthes 1985).

Barthes, Roland (7 juin 1974): L’aventure sémiologique. Le Monde, 28.

7. C'est dans cette perspective que Michael Riffaterre définit la signifiance comme «une praxis de la transformation par le lecteur», qu'il oppose au sens mimétique (Riffaterre 1983: 25).

8. Nous empruntons l'expression forme-sens d'Henri Meschonnic (1970-1977), mais un Saussure méconnu l'avait employée avant lui dans une affirmation déterminante: «Il est faux (et impraticable) d'opposer la forme et le sens. Ce qui est juste en revanche c'est d'opposer la figure vocale, d'une part, et la forme-sens de l'autre» (Saussure 1891/2002: 15).

9. Par delà certaines contradictions implicites entre Lacan et Barthes maintes fois invoquées par la critique, notre propos ici n'est que de cerner notre approche du signifiant en l'appuyant sur des formalisations connexes, à savoir: l'incomplétude ou infinitude de celui-ci, d'après Barthes, et l'ouverture à l'infini par rapport à la béance, d'après la conception lacanienne.

10. Adjectif dérivé du néologisme derridien différance.

11. Pour une approche nuancée des correspondances entre les triades de Peirce et de Lacan, nous renvoyons à Balat (2000: 37-68) et à Santaella (1986: 25-30). Pour la correspondance entre le Representamen chez Peirce et le Signifiant chez Lacan, voir aussi Balat (2000: 17-36).

12. C'est en vertu de ce principe d'implication que Jakobson (1965: 26-27) en vint à contester le fondement saussurien de l'arbitraire du signe en affirmant que conventionalité et motivation sont des caractéristiques des signes linguistiques complémentaires en non pas contradictoires.

13. On touche, là aussi, au problème de la diachronie: la diachronie sue et la diachronie non-sue, ce qui conduirait à s'interroger sur la tendance aux jeux de langage qu'ont les étymons et sur les procédés par lesquels certaines étymologies populaires parviennent à neutraliser des étymons réels. Sans doute le traçage de tels parcours sémiosiques contribuerait à appréhender certains processus d'ordre traductologique.

14. Le contexte est dynamique, le terme lui-même est sujet à équivoques. Selon trois types de référentiation, Didier Coste en distingue trois niveaux: le co-texte, l'intertexte et l'environnement ou contexte extratextuel. La mouvance du texte et du sens relève en quelque sorte de ce flottement du contexte (Coste 2003: 107-120).

15. Puig, Manuel (1976/1988): El beso de la mujer araña. Barcelona: Seix Barral.

16. Puig, Manuel (1979/1996): Le baiser de la femme-araignée. (Traduit par Albert Bensoussan) Paris: Éditions du Seuil. 
17. Pour une vue d'ensemble de la richesse et de la complexité primordiales de El beso de la mujer araña, nous renvoyons à l'édition critique établie par José Amícola et Jorge Panesi (Puig 1976/2002) pour Galaxia Gutenberg.

Puig, Manuel (1976/2002): El beso de la mujer araña. José Amícola et Jorge Panesi, dir. Coll. Archivo, 42. Nanterre: Signatarios del Acuerdo Archivos, ALLCA XX, Université Paris X.

18. Le ClÉzio, Jean-Marie Gustave (1970): La Guerre. Paris: Gallimard.

19. Le Clézio, Jean-Marie Gustave (1970/1972): La Guerra. (Traduit par Rodolfo Hinostroza) Barcelone: Seix Barral.

20. Pour une vue plus détaillée sur la question de l'iconicité en traduction, nous renvoyons à D'Asprer (2007; 2011; 2012).

21. Le Clézio, Jean-Marie Gustave (1963): Le Procès verbal. Paris: Gallimard.

Le Clézio, Jean-Marie Gustave (1973): Les Géants. Paris: Gallimard.

\section{RÉFÉRENCES}

Aristote (n. d./1966): Organon: I - Catégories; II - De l'interprétation. (Traduit par Jules Tricot 1936). Paris: J. Vrin.

Audet, Noël (1978) : Langage poétique: écart ou errance du sens. Voix et Images. 3(3):459-466.

D'Asprer, Núria (2003): Pour une lecture-traduisante ou comment faire avec la fragilité des signes: Iconicité dans La Guerre de J.-M. Le Clézio. Quaderns de traducció. 9:93-105.

D’Asprer, Núria (2007) : Cubism in translation and cubist iconicity. In: Gian Paolo GiUdicETTI et Costantino Maeder. Divagations iconiques. Iconic Divagations. Deuxième volume du troisième colloque sur l'Iconicité en langue et littérature (Université catholique de Louvain, Louvain-la-Neuve, 2003). Louvain-la-Neuve: Université catholique de Louvain - Centre d'études italiennes, 77-102.

D’Asprer, Núria (2011) : Iconicité, discontinuité et digression. Zagadnienia Rodzajów Literackich [Les Problèmes des genres littéraires]. LIV(107-108):45-67.

D’Asprer, Núria (2012): Traduire Paul Nougé: esquisse d'une pratique agissante. Hermeneus - Revista de Traducción e Interpretación. 14:79-101.

Balat, Michel (2000): Des fondements sémiotiques de la psychanalyse: Peirce après Freud et Lacan. Paris: L'Harmattan.

Barthes, Roland (1970): S/Z. Paris: Édition du Seuil.

BARThes, Roland (1982): L'Obvie et l'Obtus: Essais critiques III. Paris: Éditions du Seuil.

BARthes, Roland (1985) : L'aventure sémiologique. Paris: Éditions du Seuil.

Bensoussan, Albert (1991): El traductor en la noche oscura del sentido. In: María Luisa DONAIRE et Francisco LAFARgA, dir. Traducción y adaptación cultural: España-Francia. Oviedo: Universidad de Oviedo, 15-20.

Berman, Antoine (1984): L'épreuve de l'étranger. Paris: Gallimard.

Berman, Antoine (1985): Les tours de Babel: essais sur la traduction. Mauvezin: Trans-EuropRepress.

BrogaARD, Berit (2007): That May Be Jupiter: A Heuristic for Thinking Two-Dimensionally. American Philosophical Quarterly. (4):315-328.

Chalmers, David J. (2006): The Foundations of Two-Dimensional Semantics. In: Manuel García Carpintero et Josep Macíà, dir. Two Dimensional Semantics. Oxford/New York: Oxford University Press/Clarendon Press.

Coseriu, Eugenio (1977) : Lo erróneo y lo acertado en la teoría de la traducción. In: Eugenio Coseriu. El hombre y su lenguaje: Estudios de teoría y metodología lingüística. (Traduit par Marcos Martínez Hernández) Madrid: Gredos, 214-239.

Coste, Didier (2003): Proceso representacional y universos de referencia: de la experiencia estética a la validación empírica de descripciones. In: Ana Testa et Leticia Minhot, dir. Representación en Ciencia y en Arte. Córdoba: Editorial Brujas, 107-120.

Deleuze, Gilles (1969): Logique du sens. Paris: Les Éditions de Minuit.

Dewey, John (1946): Peirce's Theory of Linguistic Signs, Thought, and Meaning. Journal of Philosophy. 43(4):85-95. 
Ducrot, Oswald (1984): Le dire et le dit. Paris: Les Éditions de Minuit.

Ducrot, Oswald et Anscombre, Jean-Claude (1983) : L'argumentation dans la langue. Sprimont: Mardaga.

Eco, Umberto (1988): Le signe. Bruxelles: Labor.

Frege, Gottlob (1891/1971): Écrits logiques et philosophiques. (Traduit par Claude ImBERT) Coll. L'ordre philosophique. Paris: Éditions du Seuil.

GARELLI, Jacques (1991): Rythmes et mondes. Grenoble: Million.

HÉвERT, Louis (2006): Les structures du signe. Le signe selon Klinkenberg. Signo. Site Internet de théorie sémiotique. Consulté le 19 avril 2014, <http://www.signosemio.com/klinkenberg/ signe.asp>.

JаковSON, Roman (1959): On Linguistic Aspects of Translation. In: Reuben Arthur Brower, dir. On Translation. Cambridge: Harvard University Press, 232-239.

Jakobson, Roman (1959/1963): Essais de linguistique générale. Paris: Les Éditions de Minuit.

Jаковson, Roman (1965): À la recherche de l'essence du langage. Problèmes du langage. Diogène $n^{\circ}$ 51. Paris: Gallimard, 22-38.

LACAN, Jacques (1954/1975) : La fonction créatrice de la parole. In: Les écrits techniques de Freud (Séminaire I, 1953-1954/1975). Paris: Éditions du Seuil, 361-374.

LACAN, Jacques (1966) : Subversion du sujet et dialectique du désir. In: Écrits II. Paris: Éditions du Seuil.

Meschonnic, Henri (1970-1977): Pour la poétique I-IV. Paris: Gallimard.

Peirce, Charles Sanders (1897/1978): Écrits sur le signe. (Traduit et commenté par Gérard Deledalle) Paris: Éditions du Seuil.

Pottien, Bernard (2007): La traduction, une affaire de 'sens'. Tinkuy. 4:37-40.

Рyм, Anthony (2010): Exploring Translation Theories. London/New York: Routledge.

RASTIER, François (1989): Sens et textualité. Paris: Hachette.

Rastier, François (1990): La triade sémiotique, le trivium, et la sémantique linguistique. Nouveaux actes sémiotiques. 9:5-39.

RAstier, François (2006) : La traduction: interprétation et genèse du sens. In: Marianne LedeRER et Centre de recherche en traductologie (Paris), dir. Le Sens en traduction. Caen : Lettres modernes Minard, 37-49.

Ricceur, Paul (1975): La métaphore vive. Paris: Éditions du Seuil.

Ricceur, Paul (2004): Sur la traduction. Paris: Bayard.

Riffaterre, Michael (1983): Sémiotique de la poésie. Paris: Éditions du Seuil.

Russell, Bertrand (1950): Logical Positivism. Revue Internationale de Philosophie. IV:1-18.

SAntaella, Lucia (1986): As três categorias peircianas e os três registros lacanianos. Cruzeiro Semiótico. 4:25-30.

SAussure, Ferdinand de (1891/2002): Écrits de linguistique générale. Texte établi et édité par Simon Bouquet et Rudolf Engler. Paris: Gallimard.

Saussure, Ferdinand de (1916/1985): Cours de linguistique générale. Annotations et commentaires de Tullio De Mauro (1976). Charles Bailly, Albert Séchehaye et Albert Riedlinger, dir. Lausanne/Paris: Payot.

Sperber, Dan et Wilson, Deirdre (1989): La pertinence: Communication et cognition. Paris: Les Éditions de Minuit.

Stecconi, Ubaldo (1994): Peirce's Semiotics for Translation. Koiné. Annali della Scuola Superiore per Interpreti e Traduttori «San Pellegrino». IV:161-180.

STECCONI, Ubaldo (2001) : Un mapa de la semiótica y sus aplicaciones a los estudios de traducción. Tonos digital. Revista electrónica de estudios filológicos. 2. Consulté le 21 mars 2011, $<$ http://www.um.es/tonosdigital/znum2/estudios/StecconiTonos2B.htm>.

TABER, Charles R. (1972): Traduire le sens, traduire le style. Langages. 7(28):53-63.

VERHESEN, Fernand (2003) : À la lisière des mots: sur la traduction poétique. Bruxelles: La Lettre Volée.

Wittgenstein, Ludwig (1953/2004): Recherches philosophiques. Paris: Gallimard. 